\title{
Diseño de un itinerario aumentado e interdisciplinar para la formación de maestros de educación primaria
}

\author{
Design of an augmented and interdisciplinary itinerary for primary education \\ teacher training
}

\begin{abstract}
María Belén San Pedro Veledo; pedromaria@uniovi.es | (iD https://orcid.org/0000-0002-1038-5747
Lourdes Villalustre Martínez; villalustrelourdes@uniovi.es | iD https://orcid.org/0000-0002-5427-5355

Mónica Herrero Vázquez; herreromonica@uniovi.es | (iD) https://orcid.org/0000-0001-8922-0189
\end{abstract}

Universidad de Oviedo (España)

\section{Resumen}

La Realidad aumentada (RA) configura un nuevo ambiente de aprendizaje más enriquecido, combinando contenidos físicos y digitales. Por ello, el objetivo de este trabajo se dirige al diseño de un itinerario didáctico interdisciplinar con RA destinado a estudiantes universitarios del Grado de Maestro en Educación Primaria. Así, haciendo uso de una estructura gamificada se presenta a los estudiantes un misterio que deben resolver a través de diferentes pistas y pruebas geolocalizadas de manera colaborativa e innovadora. Con todo, el itinerario propuesto pretende servir de guía a los futuros maestros/as de Educación Primaria como vehículo para promover la integración efectiva de las nuevas tecnologías y que dé respuesta a las necesidades de un alumnado cada vez más digitalizado.

Palabras clave: itinerario, realidad aumentada, gamificación, maestros en formación, interdisciplinariedad

\section{Abstract}

Augmented Reality (RA) configures a new, more enriched learning environment, combining physical and digital content. For this reason, the objective of this work is directed to the design of an interdisciplinary didactic itinerary with RA destined to BA students of Primary Education. Thus, using a gamified structure, the students face a mystery that they must solve through different tracks and geolocalized tests in a collaborative and innovative way. Thereby, the proposed itinerary aims to serve as a guide for future teachers of primary education as a vehicle to promote the effective integration of new technologies and to respond to the needs of increasingly digitalized students.

Keywords: itinerary, augmented reality, gamification, teachers in training, interdisciplinarity 


\section{INTRODUCCIÓN / MARCO TEÓRICO}

Las tecnologías móviles están redefiniendo los espacios de aprendizaje, más allá de las aulas convencionales, permitiendo desarrollar prácticas formativas en cualquier momento y lugar gracias la flexibilidad que les confieren (Villalustre y Del Moral, 2016), dando lugar al denominado aprendizaje ubicuo. Éste permite configurar un nuevo ambiente de aprendizaje más enriquecido gracias a la realidad aumentada (RA) y la geolocalización, al combinar los contenidos físicos con los digitales (Billinghurst y Duenser, 2012). El aprendizaje ubicuo contribuye a la adquisición de nuevos conocimientos al interaccionar con determinados objetos, tanto físicos como virtuales, a través de los cuales se obtiene información relevante. El uso de estas tecnologías móviles aporta cierto grado de innovación despertando la motivación e implicación de los estudiantes, tal y como apuntan Di Serio, Ibáñez y Kloos (2013). No en vano, según el INE (2015) el 95\% de los estudiantes usan ordenador habitualmente y el $65 \%$ dispone de dispositivo móvil.

En este sentido, la UNESCO (2011) establece que tanto los programas de desarrollo profesional para docentes en ejercicio, como los de formación inicial para futuros maestros deben comprender experiencias formativas enriquecidas con TIC, que los prepare para desempeñar un papel esencial en la capacitación tecnológica de sus estudiantes, ofreciendo experiencias de aprendizaje enriquecidas con nuevas tecnologías. Si bien, el estudio de la European Commission/ EACEA/ Eurydice (2015) pone de manifiesto la carencia de formación y la necesidad de capacitación de los docentes en materia TIC, pues evidencia que menos del 40\% del profesorado utiliza las TIC en sus aulas, señalando como principales obstáculos la falta de ordenadores, la limitada conexión a Internet, la escasez de software apropiado, y la falta de formación específica en la explotación didáctica de nuevas tecnologías.

Algunos estudios demuestran los beneficios del uso de TIC en el alumnado de Educación Primaria. Ching (2009) afirma que tras implementarse el proyecto Technology Foundation Standards for Students of the International Society for Technology in Education (ISTE) con 1273 estudiantes de Educación Primaria, divididos en grupos control y experimental, los estudiantes de este último obtuvieron mayores puntuaciones en inglés, matemáticas, ciencias, estudios sociales y arte. Lambrinos y Asiklari (2014) llevaron a cabo una experiencia con estudiantes de Educación Primaria en Grecia, utilizando alta tecnología en una búsqueda del tesoro. Los autores señalan que el uso de la misma es factible en estudiantes de esta etapa, implementándose fácilmente si se realiza a través de la interdisciplinariedad. Por lo tanto, es necesario habilitar a los estudiantes del Grado de Maestro en Educación Primaria, por un lado, en el uso y manejo de las TIC, y por otro, capacitarlos en el uso de conocimientos que puedan unificar de modo transversal para transmitirlos a su futuro alumnado.

Por otro lado, el modelo tradicional de enseñanza basado en la transmisión- recepción entre profesor y alumno, traslada una visión de la ciencia como conjunto de conocimientos cerrados, absolutos y definitivos, que llegan al aula a través de la transmisión fiel que hace el docente del libro de texto (Ruiz Ortega, 2007), cuando esa visión se opone a la propia naturaleza del conocimiento científico y la forma en que este se construye. Un modelo alternativo propuesto, 
al que se le atribuyen ventajas sobre el tradicional, se apoya en la investigación realizada por el alumnado (Gil Flores, 2014). El Informe ENCIENDE (Confederación de las Sociedades Científicas de España, COSCE 2011) ya afirma que la enseñanza de las ciencias debe incluir actividades y tareas que demanden del alumnado la aplicación de los conocimientos, reclamando el uso de materiales didácticos innovadores. El mismo informe señala la necesidad de un replanteamiento de la formación del profesorado de ciencias en consonancia con la renovación que se persigue, poniendo el acento en la aplicación de los contenidos en contextos diversos y relevantes. Así pues, y siguiendo esta línea, esta propuesta plantea que los estudiantes del Grado de Maestro en Educación Primaria pasen de ser usuarios pasivos de recursos educativos a creadores y productores de materiales didácticos innovadores con competencia en nuevas tecnologías, en consonancia con otros autores (Barroso y Gallego, 2017).

Aunque se reconoce la potencialidad de la RA en la educación formal como componente clave en futuros entornos de aprendizaje, todavía se sabe poco sobre cómo esta tecnología puede apoyar los procesos de enseñanza y aprendizaje en grupos de escolares de Primaria. Se ha publicado que al analizar el diálogo docente-estudiante en un estudio comparativo entre el uso de RA con otros métodos tradicionales para la enseñanza de las ciencias, se observó que los niños de 10 años que usaron RA participaban menos que los que utilizaron otros recursos como el juego de roles (role playing) (Kerawalla, Luckin, Seljeflot y Woolard, 2006). Cuando fueron entrevistados, los docentes, reconociendo los beneficios de esta tecnología, indicaron que les gustaría que estos recursos fueran más flexibles y controlables, de forma que ellos pudiesen añadir o separar distintos elementos, acortar o parar distintas secuencias. Los mismos autores sugieren algunos aspectos a considerar para adaptar con éxito la RA en la práctica de aula: contenidos flexibles que el docente pueda adaptar a las necesidades de los niños; exploración guiada para que se maximicen las oportunidades de aprendizaje en tiempo limitado y adecuación curricular. Por tanto, estos resultados también apuntan hacia el interés de que los propios docentes sean capaces de convertirse en creadores de sus propios recursos de RA para adaptarlos de forma flexible en sus aulas.

Un itinerario didáctico constituye un recorrido preestablecido, en el que se diseñan diferentes paradas, que puede realizarse en un entorno natural o urbano, presentando un gran potencial transversal e interdisciplinar para integrar las diferentes materias curriculares (Quintana, Haro, Márquez, y Fernández, 2017). A pesar de los múltiples beneficios del aprendizaje fuera el aula, aún hoy la formación de los futuros docentes apenas propicia la utilización del medio y el entorno cercano como recurso didáctico, siendo aún escasas las propuestas de itinerarios interdisciplinares para el alumnado (Quintana, Haro, Márquez, y Fernández, 2017).

En este sentido, cobra importancia el concepto de Educational Leisure Settings (ELS), espacios de ocio en los que se pueden incluir museos, rutas turísticas, yacimientos arqueológicos y lugares históricos, con un enorme potencial didáctico. El aprendizaje en estos ELS confluye en una experiencia que aúna el entretenimiento y lo educativo (Packer, 2004). La labor de los centros escolares debe ser la de coordinar experiencias educativas y de ocio como una nueva forma de adquisición de conocimientos aprovechando el aprendizaje en contextos no-formales (Fleming, Allen y Barcelona, 2011). Este tipo de actividades que vinculan lo lúdico y lo educativo pueden relacionarse con el término gamificación. Las dinámicas de juego se han incorporado a 
la educación ordinaria estimulando el aprendizaje a través de la resolución de retos (Rivero, 2017), obteniendo mayores resultados si éstos siguen una progresión.

A modo de ejemplo para destacar la potencialidad de la RA, y el interés de los futuros docentes en ser competentes en nuevas tecnologías, algunas escuelas de Primaria ya han desarrollado proyectos interesantes (Leiva y Moreno, 2015). Entre otros destacan el proyecto de QRcodificación de las calles de Rubí (Barcelona), que recoge información detallada de las calles del municipio a partir de la generación de códigos QR de las URL de los blogs creados por los alumnos de Primaria de los colegios de la zona; o el proyecto de la Península de la Magdalena, creado por el maestro con sus alumnos de Primaria, para la creación de una guía visual y ampliada de la península de la Magdalena (Leiva y Moreno, 2015).

Con todo, desde las asignaturas de Desarrollo Curricular de las Ciencias Sociales, Didáctica de las Ciencias Sociales, Didáctica del Medio Natural y su Implicación Cultural, y Tecnologías de la Información y Comunicación aplicadas a la Educación del Grado de Maestro en Educación Primaria de la Universidad de Oviedo, se presenta a los estudiantes universitarios una práctica formativa interdisciplinar destinada a, no sólo abordar contenidos específicos de cada materia, sino también a ofrecer un modelo de integración didáctica de nuevos recursos tecnológicos, en este caso, la RA. De igual modo, el itinerario propuesto pretende servir de guía a los futuros maestros/as de Educación Primaria como vehículo para promover la integración efectiva de las nuevas tecnologías que dé respuesta a las necesidades de un alumnado cada vez más digitalizado.

\section{OBJETIVOS}

- Diseñar un itinerario didáctico interdisciplinar y gamificado con RA para potenciar el aprendizaje ubicuo y el desarrollo de materiales didácticos innovadores.

- Potenciar la interdisciplinariedad en la Educación Superior, con la participación del profesorado de distintas áreas de conocimiento y los estudiantes de distintas asignaturas para el desarrollo de una propuesta educativa integradora.

- Favorecer el trabajo colaborativo entre los futuros docentes a partir de la propuesta de una experiencia basada en juegos en un contexto formativo.

\section{METODOLOGÍA}

El presente trabajo se centra en el diseño de un itinerario didáctico orientado a incrementar la motivación y la adquisición de nuevos conocimientos en los estudiantes universitarios mediante la propuesta de experiencias gamificadas. A través de la gamificación se han diseñado actividades de aprendizaje ingeniosas y atractivas que promueven la resolución de tareas de forma innovadora y colaborativa (Lee y Hammer, 2011). Con la adopción de los elementos inherentes al juego se persigue potenciar el engagement -enganche, traducción literal del inglés- o implicación de los discentes en su proceso de aprendizaje (Hanus y Fox, 2015). 
En los últimos años se está reconociendo el potencial que tiene la RA para captar la atención y motivar al alumnado, permitiéndole explorar diversos elementos del patrimonio natural y cultural que pueden ser ya inexistentes o encontrarse en mal estado de conservación, pero que pueden reconstruirse de forma virtual, como se propone en este trabajo. Por tanto la RA está emergiendo en las escuelas como un recurso particularmente útil para la enseñanza de contenidos que los estudiantes no podrían experimentar de primera mano. Sin embargo, a la vez, se reconoce la dificultad de implementar en el aula estos recursos para maximizar los aprendizajes, novedosos aún para los docentes en activo, frente a su destreza en el uso de recursos tradicionales. Para conseguir aprendizajes efectivos con esta nueva tecnología es necesario conocer qué estrategias didácticas son las más adecuadas para su implementación, y capacitar a los futuros docentes para guiar los aprendizajes interactuando entre el mundo virtual y el real. Así pues, el papel del docente resulta de nuevo clave para integrar esta tecnología en la educación formal, desde el punto de vista organizativo y didáctico, lo que requiere especial atención en los programas universitarios de formación del profesorado.

De este modo, en este trabajo se diseña un itinerario con RA en el que se incorporan los elementos propios que definen un contexto de juego, es decir, se adoptan las claves de un sistema gamificado. En concreto, se les presenta a los estudiantes como reto o desafío la resolución de un misterio que deberán resolver a través de diferentes pistas y pruebas repartidas por determinados lugares de la ciudad de Oviedo (Asturias, España). Para ello, se hace uso de diferentes herramientas de RA, en concreto:

- Códigos QR; código de barras bidimensional que puede almacenar diferentes datos codificados. Siendo utilizado en el itinerario para dar acceso a un video con un tráiler, a modo de presentación del desafío.

- Eduloc; herramienta basada en la geolocalización mediante la utilización de dispositivos móviles, que permite crear diferentes itinerarios a modo de juego. En la presente propuesta se hace uso de la opción "juego de pistas" para guiar a los estudiantes en el recorrido incorporando diferentes videos animados con la aplicación PhotoSpeak.

- WallaMe; aplicación para dispositivos móviles basada en la geolocalización destinada a insertar mensajes ocultos en determinados lugares, y que es utilizada para dar las pistas que permita a los discentes resolver el misterio propuesto.

- Hp Reveal; aplicación móvil que permite aumentar cualquier objeto o imagen, y que en el itinerario diseñado ofrecerá a los estudiantes información relevante para poder averiguar el misterio que esconde el Monte Naranco (Oviedo).

Para acometer el reto, y potenciar la competición propia de todo sistema gamificado, los estudiantes se agruparán en grupos de 4/5 personas, para trabajar colaborativamente y alcanzar un objetivo común. Con ello, se pretende no solo lograr su implicación, sino también fomentar la motivación hacia la actividad propuesta. 


\section{DISEÑO E IMPLEMENTACIÓN}

El diseño del itinerario supuso la realización de diferentes etapas de desarrollo, concretamente cuatro, a saber:

I. Trabajo previo de campo. En esta primera fase se visitaron los lugares concretos para conocer, interpretar, analizar y valorar los paisajes, y así establecer los Puntos de Interés Didáctico. Para ello, se tuvo en cuenta su valor patrimonial, cultural y natural. Se valoraron diferentes rutas y se optó por una que partía del entorno urbano y finalizaba en uno rural, con el fin de contrastar los diferentes tipos de paisaje de interés para las Ciencias Sociales y Naturales.

II. Análisis de seguridad. Se comprobó que dicho itinerario cumplía con los requerimientos necesarios para que fuera idóneo en términos de seguridad para los aprendizajes fuera del aula.

III. Utilización de herramientas RA. Tras determinar el recorrido a seguir se llevó a cabo una selección de las herramientas RA más adecuadas y pertinentes para desarrollar la experiencia formativa gamificada. En concreto, éstas se seleccionaron teniendo en cuenta: 1) la tipología del recurso a incorporar en las aplicaciones de RA (imágenes, videos, etc.); 2) disponibilidad de acceso a redes Wifi; 3 ) la capacidad de interacción que las aplicaciones posibilitaban; 4) la potencialidad didáctica de las herramientas para propiciar el aprendizaje ubicuo.

IV. Diseño del itinerario. Tras la planificación previa, se diseñó el itinerario gamificado con RA en el que se incluían diversas actividades y recursos teniendo en cuenta el entorno en el que se desarrollaban y el tipo de agrupamiento, tal y como recoge la tabla 1.

Tabla 1. Actividades y recursos de RA empleados en el itinerario gamificado.

\begin{tabular}{|c|c|c|c|c|c|}
\hline TA y TEM. $^{1}$ & $\begin{array}{l}\text { LUGAR } \\
\text { ENTORNO }\end{array}$ & 0 & APP RA & ENLAZA CON & ACTIVIDAD \\
\hline $\begin{array}{l}\text { P.G., S. } 1 \\
5^{\prime}\end{array}$ & Aula & & QR & $\begin{array}{l}\text { https://www.youtube.co } \\
\mathrm{m} / \text { watch?v=u2mndylkw } \\
\underline{x}\end{array}$ & Propuesta de reto \\
\hline $\begin{array}{l}\text { P.G., S. } 1 \\
15^{\prime}\end{array}$ & Aula & & Eduloc & & Pistas itinerario \\
\hline $\begin{array}{l}\text { P.G., S. } 1 \\
30^{\prime}\end{array}$ & $\begin{array}{l}\text { Santullano } \\
\text { (urbano) }\end{array}$ & & Wallame & & $\begin{array}{l}\text { Imagen } \\
\text { reconstrucción } \\
\text { interior pinturas }\end{array}$ \\
\hline $\begin{array}{l}\text { P.G., S. } 1 \\
30^{\prime}\end{array}$ & $\begin{array}{l}\text { Santullano } \\
\text { (urbano) }\end{array}$ & & Wallame & & $\begin{array}{ll}\text { Breve } & \text { texto } \\
\text { histórico } & \end{array}$ \\
\hline
\end{tabular}

${ }^{1}$ TA: Tipo de Agrupamiento. P.G.: Pequeño grupo. TEM.: Temporalización. S.: Sesión. 


\begin{tabular}{|c|c|c|c|c|}
\hline TA y TEM. $^{1}$ & $\begin{array}{l}\text { LUGAR } \\
\text { ENTORNO }\end{array}$ & APP RA & ENLAZA CON & ACTIVIDAD \\
\hline $\begin{array}{l}\text { P.G., S. } 1 \\
30^{\prime}\end{array}$ & $\begin{array}{l}\text { Foncalada } \\
\text { (urbano) }\end{array}$ & Wallame & & Reconstrucción 3d \\
\hline $\begin{array}{l}\text { P.G., S. } 1 \\
30^{\prime}\end{array}$ & $\begin{array}{l}\text { Foncalada } \\
\text { (urbano) }\end{array}$ & Wallame & & Texto epigrafía \\
\hline P.G., S. 1 & $\begin{array}{l}\text { Calle Covadonga } \\
\text { (urbano) }\end{array}$ & Wallame & $\frac{\text { https://www.youtube.co }}{\text { m/watch?v=k6ambk4nbl }}$ & $\begin{array}{ll}\text { Camino de } \\
\text { Santiago }\end{array}$ \\
\hline $15^{\prime}$ & & & $\mathrm{g}$ & \\
\hline $\begin{array}{l}\text { P.G., S. } 1 \\
30^{\prime}\end{array}$ & $\begin{array}{l}\text { Santa María del } \\
\text { Naranco } \\
\text { (rural) }\end{array}$ & Wallame & & Foto campanario \\
\hline $\begin{array}{l}\text { P.G., S. } 1 \\
30^{\prime}\end{array}$ & $\begin{array}{l}\text { Santa María del } \\
\text { Naranco } \\
\text { (rural) }\end{array}$ & & $\underline{\text { http://www.arbolapp.es }}$ & Información tejo \\
\hline $\begin{array}{l}\text { P.G., S. } 1 \\
30^{\prime}\end{array}$ & $\begin{array}{l}\text { San Miguel de } \\
\text { Lillo } \\
\text { (rural) }\end{array}$ & & $\begin{array}{l}\frac{\text { https://play.kahoot.it/\#/ }}{\text { k/1e573d58-52a0-4e32- }} \\
\text { 8570-ebd1a2c4c43f }\end{array}$ & $\begin{array}{l}\text { Actividad } \\
\text { toponimia }\end{array}$ \\
\hline $\begin{array}{l}\text { P.G., S. } 1 \\
30^{\prime}\end{array}$ & $\begin{array}{l}\text { San Miguel de } \\
\text { Lillo } \\
\text { (rural) }\end{array}$ & & $\underline{\text { http://www.arbolapp.es }}$ & $\begin{array}{l}\text { Visualización de } \\
\text { vegetación }\end{array}$ \\
\hline $\begin{array}{l}\text { P.G., S. } 1 \\
30^{\prime}\end{array}$ & $\begin{array}{l}\text { San Miguel de } \\
\text { Lillo } \\
\text { (rural) }\end{array}$ & HP Reveal & & $\begin{array}{l}\text { Mapa antiguas } \\
\text { fortificaciones }\end{array}$ \\
\hline $\begin{array}{l}\text { P.G., S. } 1 \\
30^{\prime}\end{array}$ & $\begin{array}{l}\text { San Miguel de } \\
\text { Lillo } \\
\text { (rural) }\end{array}$ & & $\begin{array}{l}\text { https://www.google.es/ } \\
\underline{\text { maps/ }}\end{array}$ & $\begin{array}{l}\text { Creación mapa } \\
\text { ubicación } \\
\text { fortificaciones }\end{array}$ \\
\hline $\begin{array}{l}\text { P.G., S. } 2 \\
60^{\prime}\end{array}$ & Aula & & & $\begin{array}{lr}\text { Búsqueda } & \text { de } \\
\text { canciones } & \text { y } \\
\text { leyendas } & \\
\end{array}$ \\
\hline $\begin{array}{l}\text { P.G., S. } 2 \\
5^{\prime}\end{array}$ & Aula & QR & $\begin{array}{l}\frac{\text { https://www.youtube.co }}{\mathrm{m} / \text { watch?v=hho59ollu3 }} \\
\underline{0}\end{array}$ & $\begin{array}{l}\text { Finalización reto } \\
\text { Alfonso ॥l }\end{array}$ \\
\hline
\end{tabular}

\section{RESULTADOS}

Desde las asignaturas de Desarrollo Curricular de las Ciencias Sociales, Didáctica de las Ciencias Sociales, Didáctica del Medio Natural y su Implicación Cultural, y Tecnologías de la Información y Comunicación aplicadas a la Educación del Grado de Maestro en Educación Primaria de la Universidad de Oviedo se presentó a los estudiantes la resolución de un itinerario didáctico creado previamente por las profesoras de dichas materias. En él los discentes debieron resolver un misterio a través de diferentes pistas y pruebas repartidas por determinados lugares de la ciudad de Oviedo (Asturias-España). Para su presentación se adoptó como estrategia un sistema gamificado con el doble objetivo de integrar los contenidos abordados en las diferentes materias implicadas, e incrementar su motivación e implicación. Así, el itinerario diseñado se 
estructuró en tres fases siguiendo la propuesta de García (2004): fase de preparación, fase de trabajo de campo y fase de síntesis.

\subsection{Fase de preparación}

La primera de ellas se inicia con la puesta en práctica de talleres dirigidos a los estudiantes, para conseguir un correcto manejo de las herramientas RA que tendrán que utilizar con posterioridad. Concretamente se desarrollaron sesiones formativas encaminadas a potenciar el manejo de las siguientes aplicaciones de RA: Eduloc, WallaMe y Hp Reveal. Así como la utilización de códigos QR con las diversas herramientas gratuitas existentes actualmente. Se dedicó, así mismo, una sesión para acercar a los estudiantes a la aplicación PhotoSpeak para la creación de videos animados. Por otro lado, los talleres prácticos no sólo estaban orientados al manejo técnico de las aplicaciones y herramientas, sino también a su explotación didáctica para potenciar diversos aprendizajes asociados a las distintas asignaturas.

De este modo, desde las áreas implicadas en el proyecto se proporcionaron al alumnado orientaciones y pautas que les permitieran relacionar y ejemplificar los contenidos tratados en el aula con las distintas etapas del recorrido, creando un espacio común para las tres asignaturas donde se recogieron materiales de consulta, no sólo relativos al manejo de las herramientas utilizadas sino también a los puntos de interés presentes en el itinerario didáctico.

\subsection{Fase de trabajo de campo}

La fase de trabajo de campo se corresponde con el desarrollo físico del itinerario. Para ello, se crearon previamente equipos de 4/5 personas con el fin de potenciar tanto la colaboración dentro del grupo como la competición con el resto de los equipos. Así, éste adopta los componentes básicos de un proceso de gamificación, a través de mecánicas y dinámicas de juego, que a continuación se detallan:

\section{a. Mecánicas de juego}

Descripción de la misión o desafío: El itinerario se articula a partir de un reto formulado por un personaje del pasado, en forma de recorrido, para averiguar un misterio oculto relacionado con el entorno de Oviedo. Así, los estudiantes debieron escanear un código QR presente en el entorno de la Facultad de Formación del Profesorado y Educación en el que visualizaron un video elaborado con la aplicación PhotoSpeak donde una estatua del Rey Alfonso II cobra vida para presentar la misión del itinerario gamificado, es decir, descubrir que misterio esconde un lugar emblemático de la ciudad de Oviedo. A través de diversas pistas se condujo a los estudiantes hacia el entorno del Monte Naranco donde descubrieron la existencia de una serie de antiguas fortificaciones. 


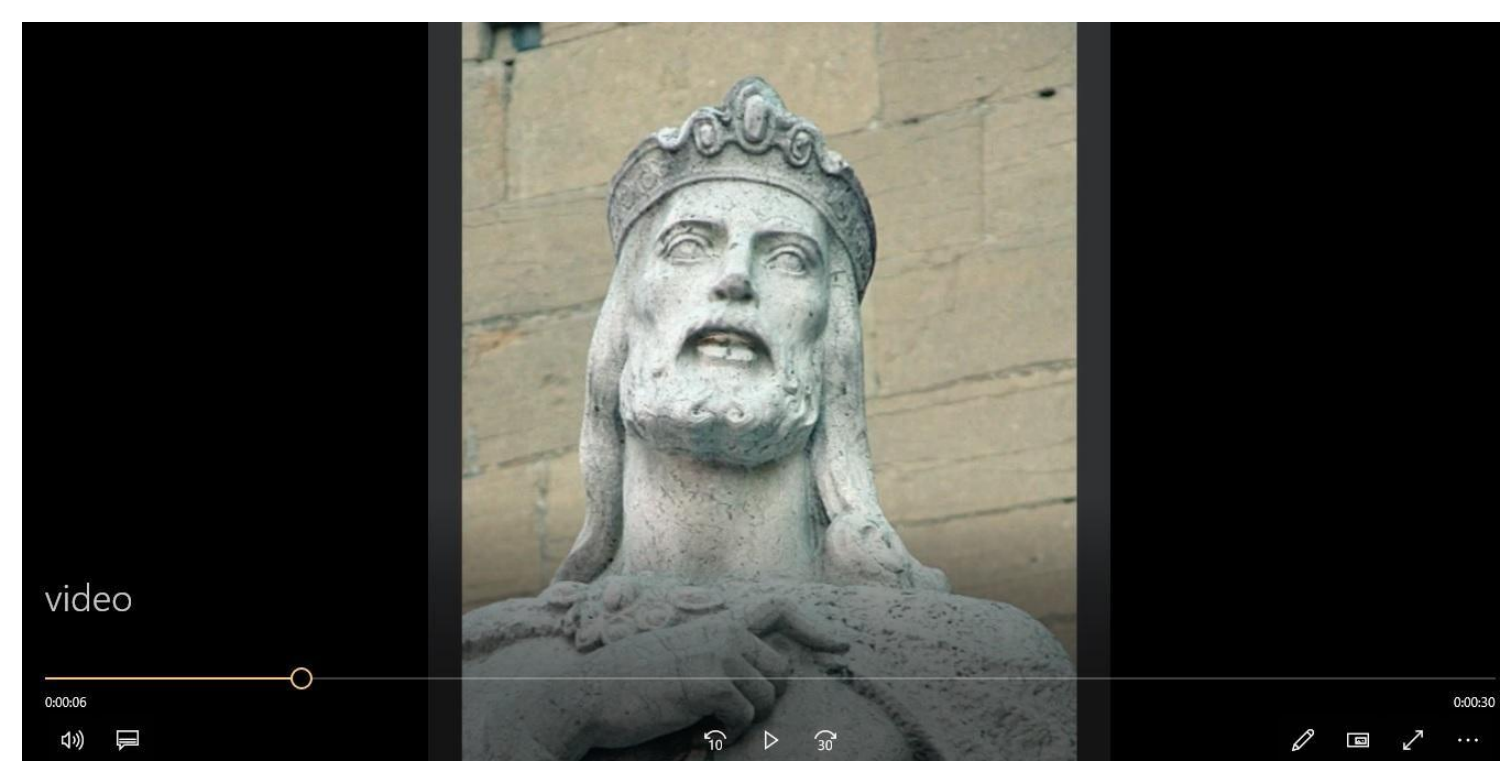

Figura 1. Captura de pantalla del vídeo de Alfonso II realizado con PhotoSpeak

Identificación de las reglas del juego: éstas se introducen a partir de un código QR al que el alumnado debe acceder para visualizar una imagen con las reglas básicas del juego iniciándose así en el itinerario gamificado. En ella, se recoge brevemente información sobre el objetivo final del juego y las normas básicas que lo rigen, en concreto se identifica que se deben seguir en todo momento las pistas suministradas sin realizar atajos, y desarrollar fair play o juego limpio, donde los equipos creados no obstaculicen o saboteen el desarrollo del resto de grupos.

Presentación de desafíos: en el itinerario gamificado se han establecido cinco puntos de interés recogidos en la aplicación Eduloc (Figura 2) y en cada uno de ellos se proporciona pistas para dirigir a los estudiantes de un punto a otro, éstos son: la iglesia prerrománica de Santullano, la fuente de Foncalada, el tramo del Camino de Santiago a su paso por la calle Covadonga, el monumento prerrománico de Santa María y San Miguel de Lillo, ambos situados en el Monte del Naranco. 


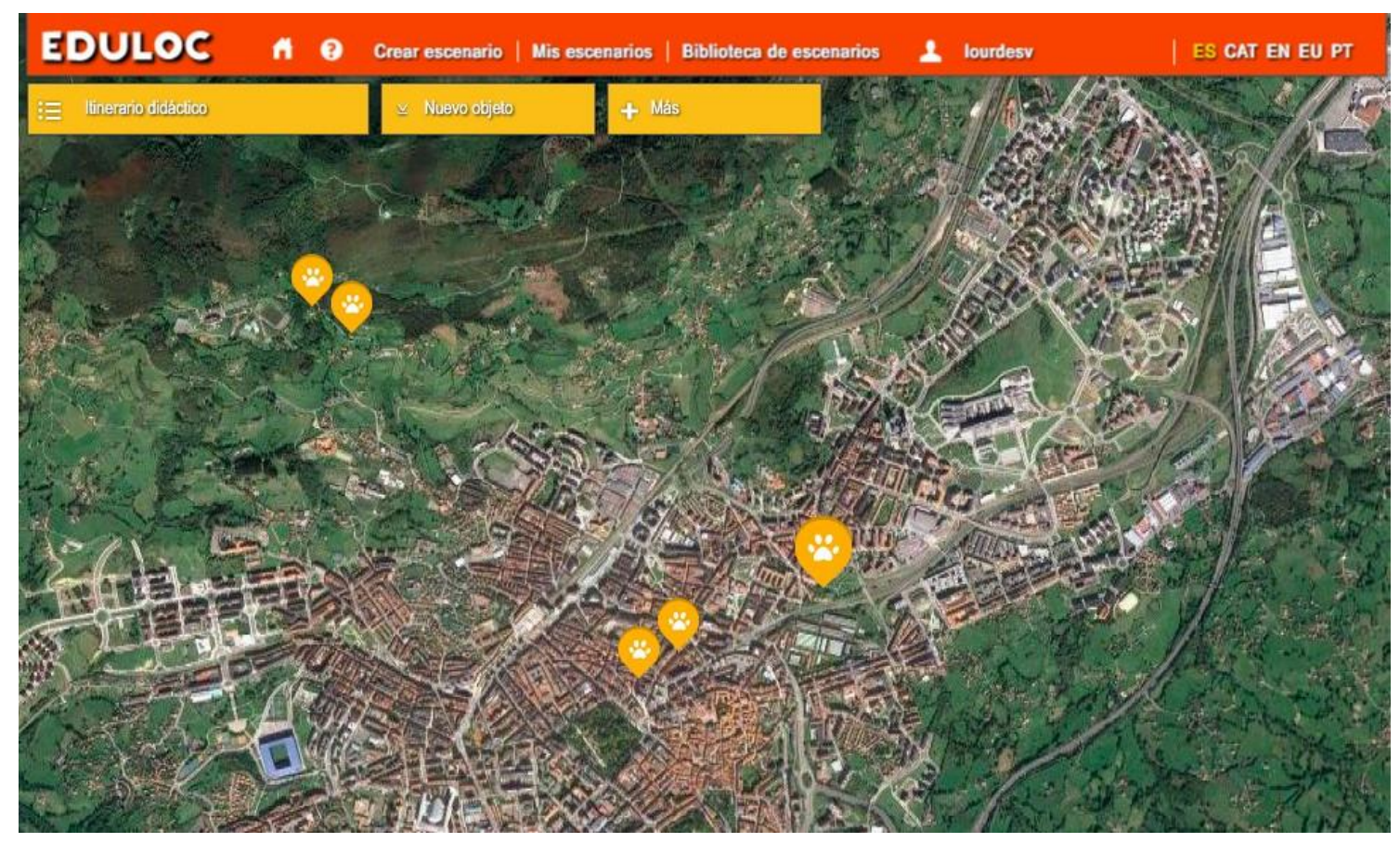

Figura 2. Juego de pistas creado con Eduloc para guiar a los estudiantes por el itinerario gamificado

En cada uno de los puntos de interés marcados en el itinerario gamificado los discentes obtienen información de diversa índole a través de diferentes aplicaciones de RA que deberán recopilar para resolver el reto final, en concreto:

- Iglesia prerrománica de Santullano; se ofrece a través de la aplicación de RA WallaMe una breve información histórica sobre el lugar, así como una imagen que recrea el esplendor de las pinturas de su interior.

- Fuente de Foncalada; nuevamente con la aplicación de RA WallaMe se añade información sobre la epigrafía del lugar y una reconstrucción en 3D de la fuente (Figura 3). 


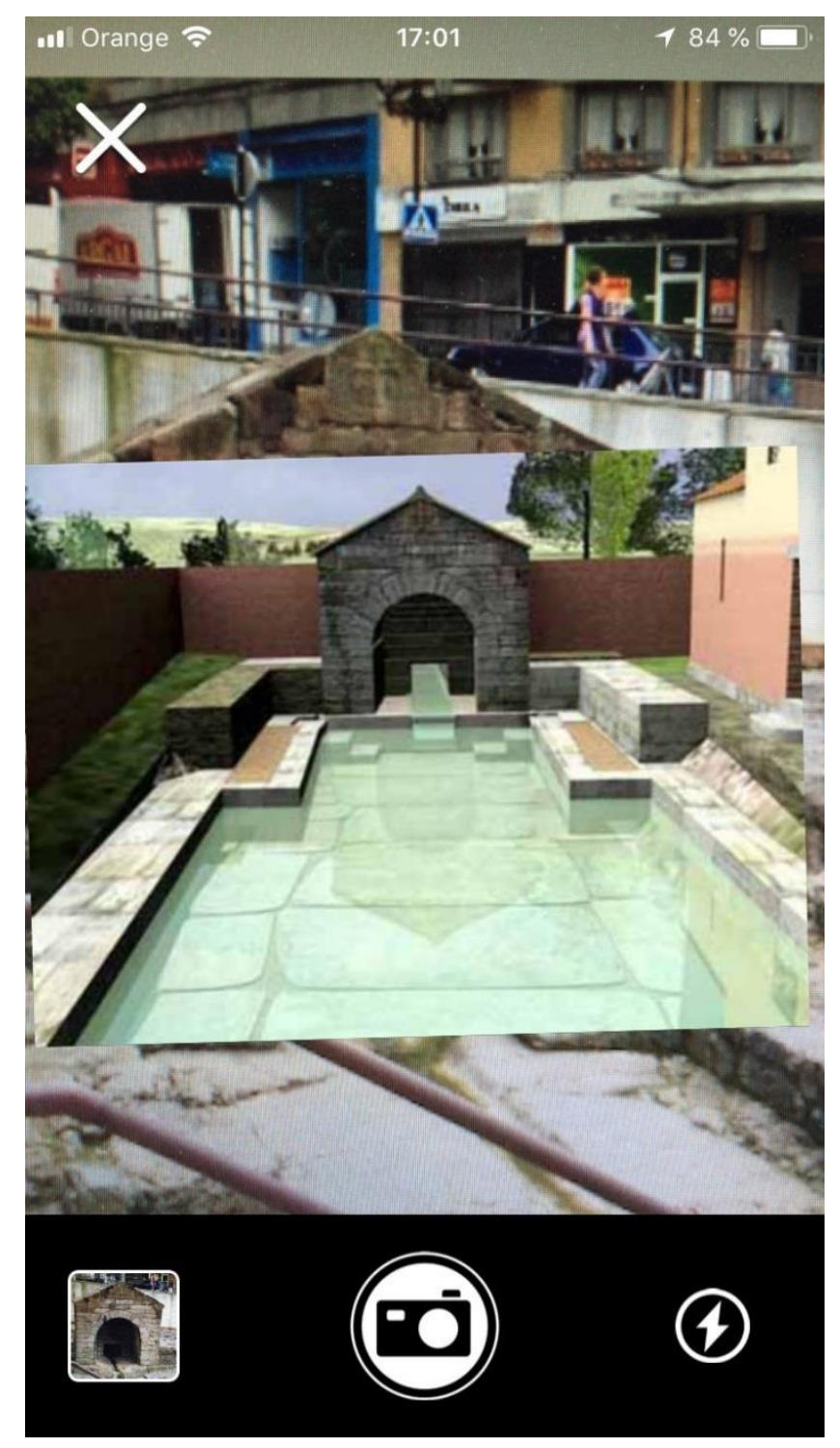

Figura 3. Recurso de geolocalización creado con WallaMe como parte del itinerario gamificado

- Camino de Santiago a su paso por la calle Covadonga; en este punto los estudiantes podrán visualizar un pequeño video sobre el Camino de Santiago a su paso por Oviedo activado a través de la aplicación de RA WallaMe.

- Monumento prerrománico de Santa María del Naranco; en esta parte final del recorrido los estudiantes deberán utilizar la aplicación Arbolapp (Figura 4) para identificar diferentes arboles de interés de la zona, así como WallaMe para obtener información relativa al tejo y acceder a una fotografía antigua donde se aprecia la existencia de un campanario añadido al monumento prerrománico. 


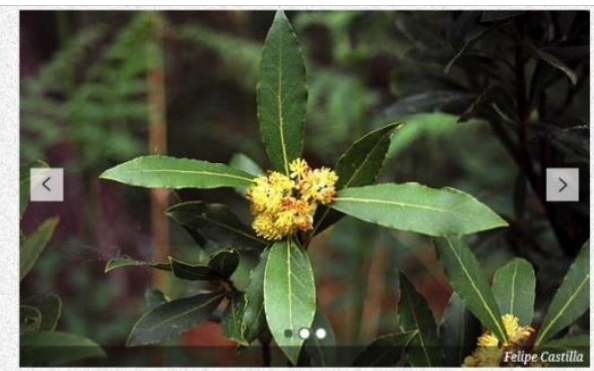

\section{Laurus nobilis \\ Laurel}

Laurel (cast.); llorer (cat)); erramua, ereñotz (eusk); loureiro (gall),; loureiro, louro (port.); bay laurel, sweet bay, bay tree, true laurel, Grecian laurel, laurel tree, laurel (ing.).

AUTÓCTONA

“¿Cuándo serás laurel, dulce tirana? / Que no te quiero yo para aceitunas, / sino para mi frente, hermosa Juanaw.

'Como suele correr desnudo atleta', Lope de Vega

\section{DESCRIPCIÓN}

El laurel es un pequeño árbol de tronco recto, corteza lisa y grisácea, que alcanza los $10 \mathrm{~m}$ de altura y que proyecta una densa sombra. Toda la planta contiene aceites esenciales que la hacen aromática, sobre todo sus hojas, que son persistentes, simples, alternas, de hasta $15 \mathrm{~cm}$ de largo, oblongo-lanceoladas, de margen entero - aunque a veces se vean un poco

Figura 4. Información extraída de la aplicación Arbolapp

- Monumento prerrománico de San Miguel de Lillo; nuevamente se hace uso de la aplicación Arbolapp para identificar los arboles de esta zona. De igual modo, los estudiantes deberán acceder a una imagen impresa de las canteras próximas a este entorno para escanearla con la aplicación Hp Reveal y visualizar una imagen con las fortificaciones existentes en el pasado en ese espacio (Figura 5).

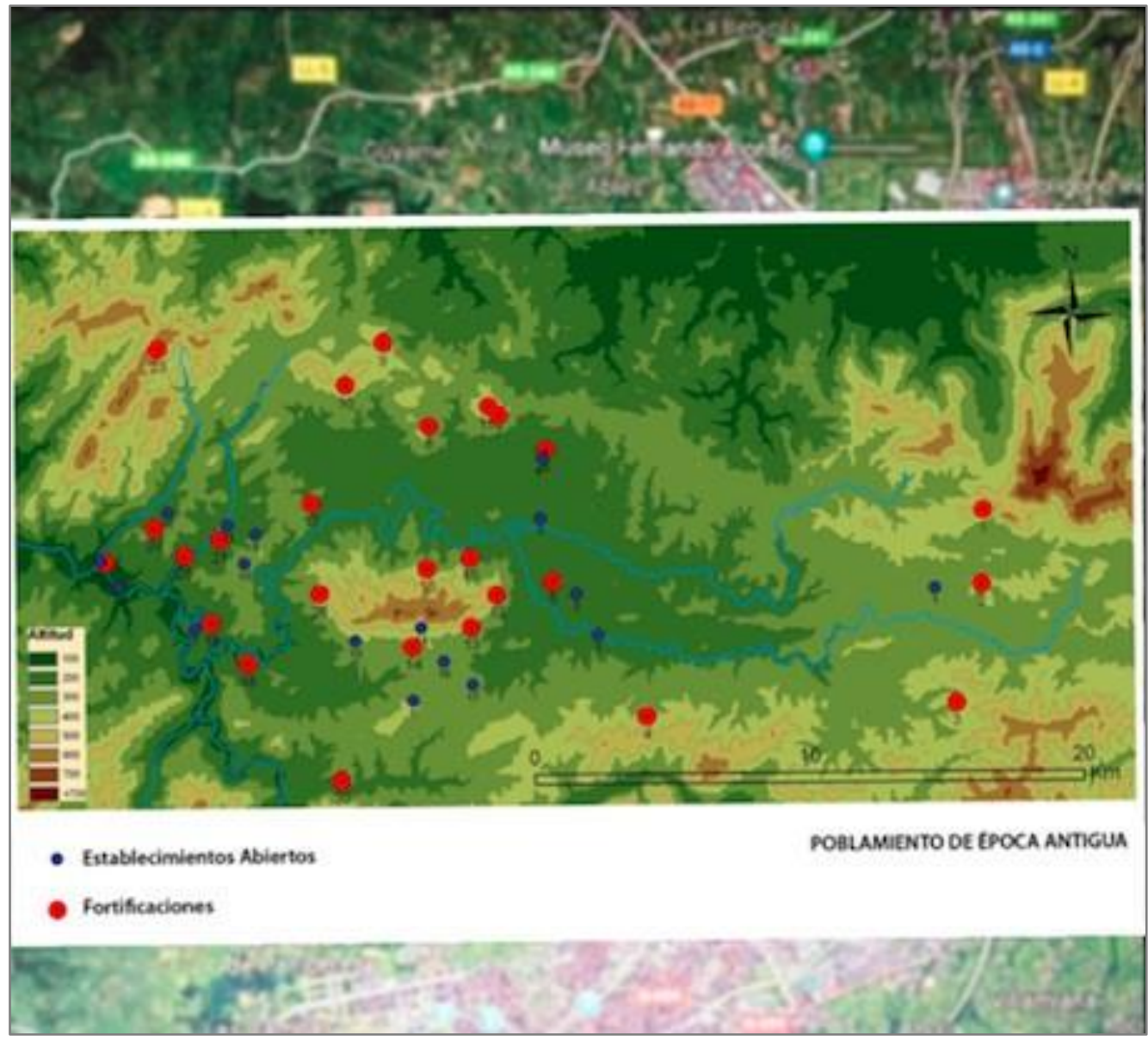

Figura 5. Recurso creado con HP Reveal para mostrar varias fortificaciones de época antigua 
De igual modo, en esta parada final los discentes deberán resolver el desafío propuesto, empleando en primer lugar la aplicación Kahoot para resolver una actividad relacionada con la toponimia del lugar, y posteriormente la herramienta Google Maps para efectuar una reconstrucción geográfica de las antiguas fortificaciones presentes en este espacio.

\section{b. Dinámicas de juego}

En relación con las dinámicas de juego, los discentes formaron grupos de 4/5 personas con el objeto de superar el reto, como ya se ha mencionado. Como condición básica todos los grupos debían finalizar el itinerario, siendo el grupo ganador aquel que consiguiera descifrar primero el misterio oculto en el Monte Naranco mediante la creación e interpretación de un mapa con la situación de varias fortificaciones de época antigua que rodeaban el mismo, algunas desaparecidas en la actualidad debido a la actividad de la industria extractiva y la expansión urbana. A la vuelta, en la Facultad de Formación del Profesorado y Educación los estudiantes escanearon otro código QR que contenía un video creado con PhotoSpeak donde nuevamente la estatua del Rey Alfonso II, a modo de cierre del juego, explicaba las consecuencias sobre el entorno que tiene la acción humana e industrial.

\subsection{Fase de síntesis}

La fase de síntesis tuvo lugar en el aula, con la realización de distintas actividades relacionadas con la RA y la geolocalización, y más específicamente se solicitó a los estudiantes la creación de recursos aumentados a partir de las aplicaciones y herramientas utilizadas en el itinerario gamificado. Con ello, se pretendía consolidar su manejo al tiempo que los futuros maestros de Educación Primaria efectuaban una explotación didáctica de las mismas.

De igual modo, los discentes desarrollaron actividades vinculadas a otras materias, encaminadas a conocer y explorar la alteración de masas arbóreas en el monte Naranco, con la ayuda de la web Global Forest Watch. Como práctica final, los estudiantes deben realizaron una propuesta de actividades vinculadas al itinerario para el alumnado de Educación Primaria, en las que la RA y la geolocalización adquirieron protagonismo como recurso formativo.

\section{DISCUSIÓN}

En este trabajo se muestran los resultados de una propuesta docente interdisciplinar, que surge de la colaboración entre el profesorado de tres áreas de conocimiento diferentes en la Facultad de Formación del Profesorado y Educación de la Universidad de Oviedo. La puesta en común de objetivos y contenidos transversales para elaborar el itinerario interdisciplinar gamificado, la selección de las herramientas y recursos tecnológicos de RA y el trabajo de campo desarrollado se ha planteado desde una metodología activa y colaborativa. Esta metodología 
desarrollada por el profesorado se traslada como propuesta didáctica conjunta a los estudiantes del Grado en Maestro en Educación Primaria, para capacitar a los futuros docentes en el desarrollo competencial necesario para su futuro desempeño profesional.

Si bien la propuesta del itinerario gamificado fue bien acogida por los estudiantes universitarios debido a su utilidad didáctica como herramienta interdisciplinar, en consonancia con lo indicado por Lambrinos y Asiklari (2014), se ha observado que es susceptible de mejoras. El planteamiento de itinerarios basados en el uso de la geolocalización puede tener algunas limitaciones técnicas derivadas de los problemas de conectividad al tener que utilizar en algunos tramos redes de pago. De igual modo, el desplazamiento de grupos numerosos afectó a la temporalización inicialmente planificada, necesitándose en algunos casos más tiempo del estimado.

Con esta experiencia, diseñada por docentes de tres asignaturas distintas de la misma titulación, se constata: por una parte, la necesidad de una mayor coordinación entre el profesorado universitario para evitar la fragmentación de la enseñanza en diferentes contextos, así como el interés de diseñar herramientas didácticas que requieran por parte de los estudiantes de la integración de contenidos, procedimientos y actitudes y valores, que hasta ahora se trataban de forma no integrada. Esta línea de trabajo iniciada, busca capacitar a los estudiantes universitarios para que diseñen sus propios itinerarios gamificados, lo que permitirá abordar una investigación no sólo basada en el uso didáctico de recursos de RA, sino en su potencialidad para la creación de recursos didácticos innovadores y creativos para potenciar aprendizajes interdisciplinares en escenarios ubicuos, tal y como apuntan Di Serio, Ibáñez y Kloos (2013).

\section{REFERENCIAS BIBLIOGRÁFICAS}

Barroso, J.M., y Gallego, O.M. (2017). Producción de recursos de aprendizaje apoyados en Realidad Aumentada por parte de estudiantes de magisterio. EDMETIC, 6(1), 23-38.

Billinghurst, M., y Duenser, A. (2012). Augmented reality in the classroom. Computer, 7, 56-63.

Ching, G. S. (2009). Implications of an experimental information technology curriculum for elementary students. Computers y Education, 53(2), 419-428. doi: 10.1016/j.compedu.2009.02.019

Confederación de Sociedades Científicas de España (COSCE) (2011). Informe ENCIENDE: Enseñanza de las Ciencias en la Didáctica escolar para edades tempranas en España. Madrid: COSCE.

Di Serio, Á., Ibáñez, M. B., y Kloos, C. D. (2013). Impact of an augmented reality system on students' motivation for a visual art course. Computers y Education, 68, 586-596 
European Commission/EACEA/Eurydice (2015). The Teaching Profession in Europe: Practices, Perceptions, and Policies. Eurydice Report. Luxembourg: Publications Office of the European Union.

Fleming, D.S., Allen, L.R., Barcelona, R.J. (2011). Back to the future: The potential relationship between leisure and education. New Directions for Youth Development, 130, 43-57. doi: $\underline{10.1002 / \mathrm{yd} .396}$

García de la Vega, A. (2014). El itinerario geográfico como recurso didáctico para la valoración del paisaje. Didáctica Geográfica, 6, 79-95.

Gil Flores, J. (2014). Metodologías didácticas empleadas en las clases de ciencias y su contribución a la explicación del rendimiento. Revista de Educación, 366, 190-214.

Hanus, M. D., y Fox, J. (2015). Assessing the effects of gamification in the classroom: A longitudinal study on intrinsic motivation, social comparison, satisfaction, effort, and academic performance. Computers y Education, 80, 152-161.

INE (2017). Encuesta sobre equipamiento y uso de tecnologías de información y comunicación en los hogares. Disponible en http://www.ine.es/prensa/tich 2017.pdf -Fecha de consulta 15/07/2018.

Kerawalla, L., Luckin, R., Seljeflot, S., y Woolard, A. (2006). "Making it real": exploring the potential of augmented reality for teaching primary school science. Virtual reality, 10(3-4), 163-174.

Lambrinos, N. y Asiklari, F. (2014). The introduction of GIS and GPS through local history teaching in primary school. European Journal of Geography, 5(1), 32-47.

Lee, J. J., y Hammer, J. (2011). Gamification in education: What, how, why bother?. Academic Exchange Quarterly, 15(2), 146-152.

Olivencia, J. J. L., y Moreno, N. M. (2015). Tecnologías de geolocalización y realidad aumentada en contextos educativos: experiencias y herramientas didácticas. DIM: Didáctica, Innovación y Multimedia, (31), 1-18.

Packer, J. M. (2004). Motivational Factors and the Experience of Learning in Educational Learning Settings (Tesis doctoral). Queensland University of Technology, Queensland. Disponible en https://eprints.qut.edu.au/15911/ -Fecha de consulta 29/05/2018.

Quintana, S. M., Haro, J. C. A., Márquez, M. M. y Fernández, J. A. L. (2017). Propuesta de itinerario interdisciplinar en la formación del profesorado de Educación Primaria en el ámbito de las Ciencias Sociales y Experimentales. Didáctica de las Ciencias Experimentales y Sociales, 31, 79-9.

Rivero, P. (2017). Procesos de gamificación en el aula de Ciencias Sociales. Íber. Didáctica de las Ciencias Sociales, Geografía e Historia, 86, 4-6. 
Ruiz Ortega, F.J. (2007) Modelos didácticos para la Enseñanza de las Ciencias Naturales. Revista Latinoamericana de Estudios Educativos (Colombia), 3(2), 41-60

UNESCO (2011). Competency Framework for teachers. París: UNESCO.

\section{Para citar este artículo:}

San Pedro Veledo, M., Villalustre Martínez, L., y Herrero Vázquez, M. (2019). Diseño de un itinerario aumentado e interdisciplinar para la formación de maestros de educación primaria. Edutec. Revista Electrónica De Tecnología Educativa, (68), 54-69. https://doi.org/10.21556/edutec.2019.68.1293 